\title{
Equivalent Circuit Analysis of Photovoltaic-Thermoelectric Hybrid Device with Different TE Module Structure
}

\author{
Haijun Chen, ${ }^{1}$ Ning Wang, ${ }^{1,2}$ and Hongcai $\mathrm{He}^{1}$ \\ ${ }^{1}$ State Key Laboratory of Electronic Thin Films and Integrated Devices and School of Microelectronics and Solid-State Electronics, \\ University of Electronic Science and Technology of China, Chengdu 610054, China \\ ${ }^{2}$ Institute of Electronic and Information Engineering, University of Electronic Science and Technology of China, \\ Dongguan 523808, China
}

Correspondence should be addressed to Ning Wang; ning_wang@uestc.edu.cn

Received 14 March 2014; Accepted 14 April 2014; Published 7 May 2014

Academic Editor: Shaohua Shen

Copyright (C) 2014 Haijun Chen et al. This is an open access article distributed under the Creative Commons Attribution License, which permits unrestricted use, distribution, and reproduction in any medium, provided the original work is properly cited.

Combining two different types of solar cells with different absorption bands into a hybrid cell is a very useful method to improve the utilization efficiency of solar energy. The experimental data of dye-sensitized solar cells (DSSCs) and thermoelectric generators (TEG) was simulated by equivalent circuit method, and some parameters of DSSCs were obtained. Then, the equivalent circuit model with the obtained parameters was used to optimize the structure design of photovoltaic- (PV-) thermoelectric (TE) hybrid devices. The output power $\left(P_{\text {out }}\right)$ first increases to a maximum and then decreases by increasing the TE prism size, and a smaller spacing between p-type prism and n-type prism of a TE p-n junction causes a higher output power of TEG and hybrid device. When the spacing between TE prisms is $15 \mu \mathrm{m}$ and the optimal base side length of TE prism is $40 \mu \mathrm{m}$, the maximum theoretical efficiency reaches $24.6 \%$ according to the equivalent circuit analysis. This work would give some enlightenment for the development of high-performance PV-TE hybrid devices.

\section{Introduction}

Dye-sensitized solar cells (DSSCs) have been widely studied since reported by O’Regan and Grätzel in 1991 [1]. They were considered as an alternative to conventional solar cells due to their low cost and high theoretical conversion efficiency $[2,3]$. In a DSSC, a mesoporous semiconductor film is sensitized with organic dye molecules which can absorb sunlight and generate excited electrons. However, typical DSSCs only absorb part of visible and near-infrared light [4] and unabsorbed sunlight wastes as heat, which is one of the main causes of the low experimental conversion efficiency of DSSCs.

In order to improve the conversion efficiency, it may be an effective method to combine two different DSSCs with different range of light absorption into a hybrid cell. Nattestad et al. proposed a novel $\mathrm{p}-\mathrm{DSSC} / \mathrm{n}$-DSSC hybrid cell, which realized a $2.42 \%$ conversion efficiency [5]. Yanagida et al. prepared DSSC (N719 dye)/DSSC (black dye) hybrid cells with a power conversion efficiency of $10.6 \%$ [6]. However, the research that two DSSCs were integrated did not achieve a satisfying high conversion efficiency. Even if for the integrated DSSCs, a considerable part of the sunlight was unabsorbed and converted into heat, so the waste heat utilization of the solar cells should be the key to improve the solar energy utilization efficiency. Actually, our previous work [7] reported a novel method to improve the solar energy conversion efficiency, which is to combine a transparent DSSC, a solar selective absorber (SSA), and a thermoelectric generator (TEG) into a hybrid device, and the novel photovoltaic-thermoelectric (PV-TE) hybrid device achieved a high conversion efficiency of $13.8 \%$ [7]. However, the problem of matching between the output current or voltage of DSSC and TEG was not taken into account, so the conversion efficiency of PV-TE hybrid device did not reach the best. In this work, the photovoltaicthermoelectric hybrid device is analyzed with an equivalent circuit method. The equivalent circuit method has often been used to analyze solar cells [8] as well as to evaluate DSSCs 
[9-11]. The relation between the conversion efficiency of the hybrid device and the internal structure of TE module is calculated based on equivalent circuit models.

\section{Modeling of Equivalent Circuit for DSSCs and TE Modules}

2.1. Modeling of Equivalent Circuit for DSSCs. This work analyzed the DSSCs by using an equivalent circuit of a onediode model shown in Figure 1(a) as described in previous reports $[11,12]$, and the equation of the equivalent circuit is written as follows [12]:

$$
I=I_{\mathrm{ph}}-I_{0}\left[\exp \left(q \frac{V+I R_{s}}{n k T}\right)-1\right]-\frac{V+I R_{s}}{R_{\mathrm{sh}}},
$$

where $I_{\mathrm{ph}}$ is the photocurrent, $I_{0}$ is the initial current, $R_{s}$ is the series resistance, $R_{\mathrm{sh}}$ is the parallel resistance, $n$ is the diode factor, $q$ is the elementary electric charge, $k$ is the Boltzmann constant, and $T$ is the temperature.

The existing experimental data [7] (AM 1.5 G, $100 \mathrm{~mW} \mathrm{~cm}^{-2}$ ) as the scattered small circles in Figure 2(a) are fitted by using formula (1) and least square method, to calculate some parameters of DSSC, and the fitting curve is shown as the solid line in Figure 2(a). Through the simulation, the values of $I_{0}, I_{\mathrm{ph}}, R_{s}, R_{\mathrm{sh}}$, and $n$ for the DSSC are obtained as $20.16 \mathrm{pA}, 1.296 \mathrm{~mA}, 32.08 \Omega, 7500 \Omega$, and 1.427 , respectively, which will be necessary for the subsequent analysis of hybrid devices.

2.2. Modeling of Equivalent Circuit for TE Modules. A thermoelectric generator (TEG) with simple structure can be regarded as a steady voltage source in series with internal resistance $[13,14]$. The equivalent circuit of the TEG was shown in Figure 1(b). The output current $(I)$ and the resistance of the TE generator $\left(R_{s \mathrm{TE}}\right)$ could be calculated using the following [13]:

$$
\begin{gathered}
I=\frac{\alpha N\left(T_{h}-T_{c}\right)-V}{R_{s \mathrm{TE}}}, \\
R_{s \mathrm{TE}}=N \times\left(\frac{L_{p}}{A_{p} \sigma_{p}}+\frac{L_{n}}{A_{n} \sigma_{n}}\right),
\end{gathered}
$$

where $\alpha$ is the Seebeck coefficient, $T_{h}$ is the hot-side temperature, $T_{c}$ is the cold-side temperature, $R_{s \mathrm{TE}}$ is the thermoelectric material resistance, $L_{p}, A_{p}, L_{n}$, and $A_{n}$, respectively, represent the length and the area of n-type thermoelectric material and the $\mathrm{p}$-type thermoelectric material, $\sigma_{p}$ and $\sigma_{n}$ are the conductivity of n-type thermoelectric material and p-type thermoelectric material, respectively, and $N$ is the number of $\mathrm{p}-\mathrm{n}$ junctions in TEG. All the TE $\mathrm{p}-\mathrm{n}$ junctions are square prisms in the same size as shown in Figure 3; that is, $L_{p}=$ $L_{n}=L$ and $A_{p}=A_{n}=A=a^{2}$, where $a$ is the base side length of p-type prism or n-type prism in a $\mathrm{p}$-n junction. Then, (3) can be simplified into (4). Consider

$$
R_{s \mathrm{TE}}=N \times \frac{L}{a^{2}}\left(\frac{1}{\sigma_{p}}+\frac{1}{\sigma_{n}}\right) .
$$

Here, the n-type and the p-type thermoelectric material stay the same; namely, $\sigma_{p}$ and $\sigma_{n}$ remain unchanged, so (4) can be further simplified to be as follows:

$$
R_{s \mathrm{TE}}=\frac{2 N L}{a^{2} \sigma_{e}}
$$

where $\sigma_{e}$ is defined as effective conductivity according to the following:

$$
\frac{1}{\sigma_{e}}=\frac{1}{\sigma_{p}}+\frac{1}{\sigma_{n}}
$$

Equations (2) and (4) are used to fit the existing experimental data by least square method to calculate some parameters of TEG as follows: $\sigma_{e}=5.25 * 10^{4} \mathrm{Sm}^{-1}$ and $\alpha=0.222 \mathrm{mV} \mathrm{K}^{-1}$, which will be necessary for the subsequent analysis of hybrid devices. The $J-V$ curves of both experimental and fitting data are shown in Figure 2(b). The fitting curve is slightly higher than the experimental curve for the reason that the fitting calculation reckons without contact resistance of the TE modules.

2.3. Equivalent Circuit Analysis of PV-TE Hybrid Devices. In the hybrid device, the DSSC and TEG are connected in series. The equivalent circuit of the hybrid device is shown in Figure 1(c). According to the nature of the series, the current and the voltage in the hybrid device comply with the following formula:

$$
\begin{gathered}
I=I_{\mathrm{DSSC}}=I_{\mathrm{TE}}, \\
V=V_{\mathrm{DSSC}}+V_{\mathrm{TE}},
\end{gathered}
$$

where $I_{\mathrm{DSSC}}$ and $I_{\mathrm{TE}}$ are the output current through the DSSC and TEG, respectively, and $V_{\mathrm{DSSC}}$ and $V_{\mathrm{TEG}}$ are the output voltage of the DSSC and TEG, respectively.

The $I-V$ relationship of the hybrid device can be expressed as (8), which can be obtained by simultaneous equations (1), (2), (4), (5), and (6). Consider

$$
I=I_{\mathrm{ph}}-I_{0}\left[\exp \left(q \frac{V-\alpha \Delta T+I R}{n k T}\right)-1\right]-\frac{V-\alpha \Delta T+I R}{R_{\mathrm{sh}}},
$$

where $\Delta T=T_{h}-T_{c}$ and $R=R_{s}+R_{s \mathrm{TE}}$.

Figure 2(c) shows the $J-V$ curves of the experimental and the fitting results of the PV-TE hybrid device, where fitting calculation ignores the wire resistance and contact resistance, and thus the fitting short-circuit current is slightly larger than the experimental result.

\section{Improvement of PV-TE Hybrid Device with Different TE Module Structure}

As for the PV-TE hybrid devices, it is difficult to improve the efficiency of DSSC and the performance of the TE materials. Relatively, changing the structure of TE modules is easy to implement. The effect of the size of the TE modules on the efficiency of the hybrid device is analyzed on the basis of the 


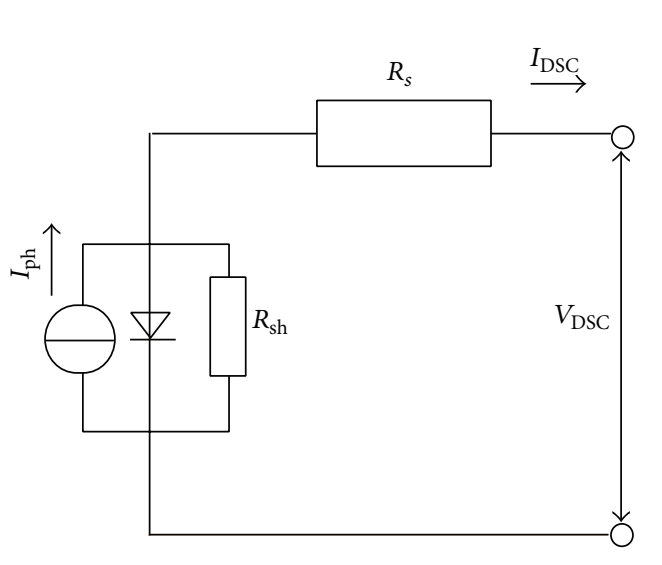

(a)

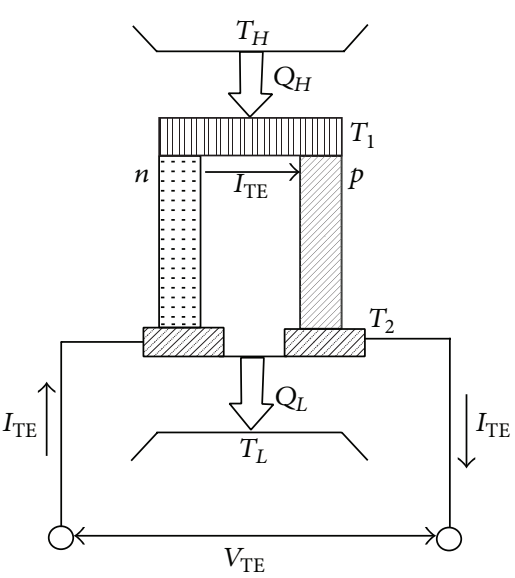

(b)

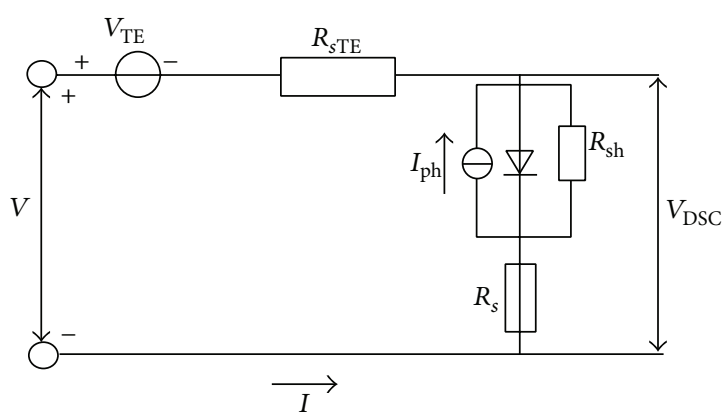

(c)

FIGURE 1: Equivalent circuit of (a) single DSSC, (b) single TE, (c) series DSSC, and TE hybrid device.

above calculated results, to determine the best size of the TE modules. The results of calculation can greatly facilitate the experimental procedure.

The above calculation is to fit the experimental data, which verified the feasibility of the calculation method and determined some parameters required in the optimization process. The following optimization calculation assumes that the temperature difference $(\Delta T)$ between the two sides of TEG is fixed at $6 \mathrm{~K}$ with the same effective gross lighting area. The optimization calculation is to change the output properties of TEG by changing the size of TE modules and to better match the outputs of DSSC. Figure 3 shows the top view of the TE $p-n$ junctions array. The number of $p-n$ junctions changes with the TE $p-n$ junction's sectional area and spacing between p-type prism and n-type prism in a TE p-n junction prism, which can be expressed as follows:

$$
N=\left[\operatorname{int}\left(\frac{\sqrt{S}-d}{a+d}\right)\right]^{2},
$$

where $S$ is the effective area of TEG (here, it is $6.25 \mathrm{~mm}^{2}$ ), $a$ is the base side length of TE prism, and $d$ is the spacing between $\mathrm{p}$-type prism and n-type prism of a TE $\mathrm{p}$-n junction, as shown in Figure 3. When $a$ or $d$ of TE p-n junction decreased and the total effective area of TEG remains unchanged, the number of $\mathrm{p}-\mathrm{n}$ junctions increases. As a result, the output power of TEG increases, but it is also accompanied with the internal resistance of TEG increasing, which would decrease the TEG's short-circuit current. The structure for the best performance of each component may not be consistent with that for the optimal efficiency of the whole hybrid device, which means that it is infeasible to get an optimal hybrid cell by simply combining an optimal DSSC and an optimal TEG. Comprehensive evaluation of these two factors is necessary to find an optimal solution of the area and space of TE $p-n$ junctions and, consequently, to obtain the optimal properties of the PV-TE hybrid devices. It is possible to further improve the efficiency of the PV-TE hybrid devices in our previous work as reported in [7] by optimizing the design of internal structure parameters in the TE modules to make a good match between the outputs of PV cells and TEG.

A series model of the PV-TE hybrid devices is designed as shown in Figure 1(c), in accordance with our previous experimental structure of hybrid devices [7]. Figure 4 shows the effects of internal structure parameters in the TE modules on the output power $\left(P_{\text {out }}\right)$ of hybrid cell with a limited total effective area. The output power $\left(P_{\text {out }}\right)$ first increases to a maximum and then decreases by increasing the TE prism size a. Figure 5 shows the $J-V$ curves of TEG, DSSC, and hybrid cell under simulated AM 1.5 solar spectrum irradiation at $100 \mathrm{~mW} \mathrm{~cm}{ }^{-2}$. When $d$ is $30 \mu \mathrm{m}$, the optimal TE prism size $a$ is $42 \mu \mathrm{m}$ and the maximum output power is $1.185 \mathrm{~mW}$ with 


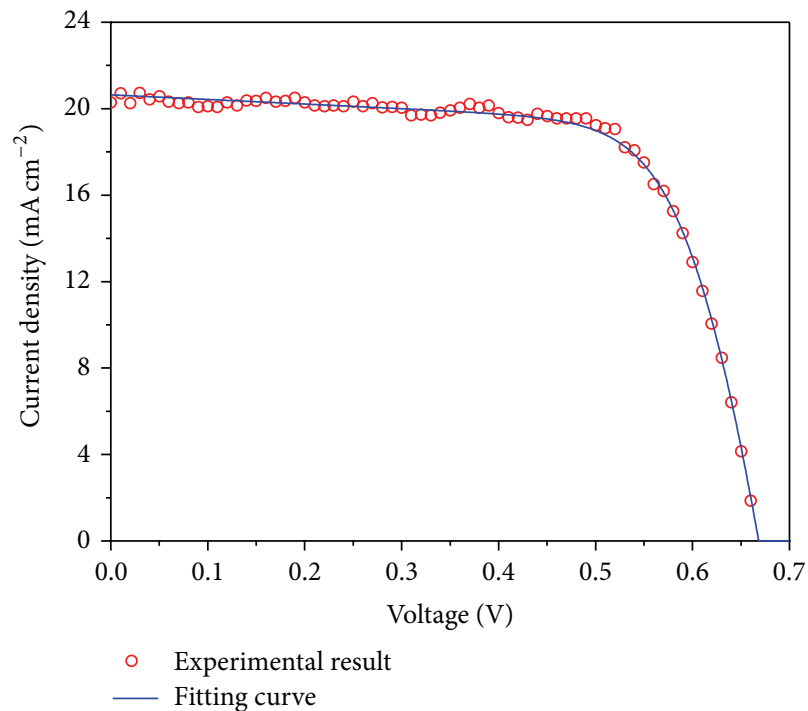

(a)

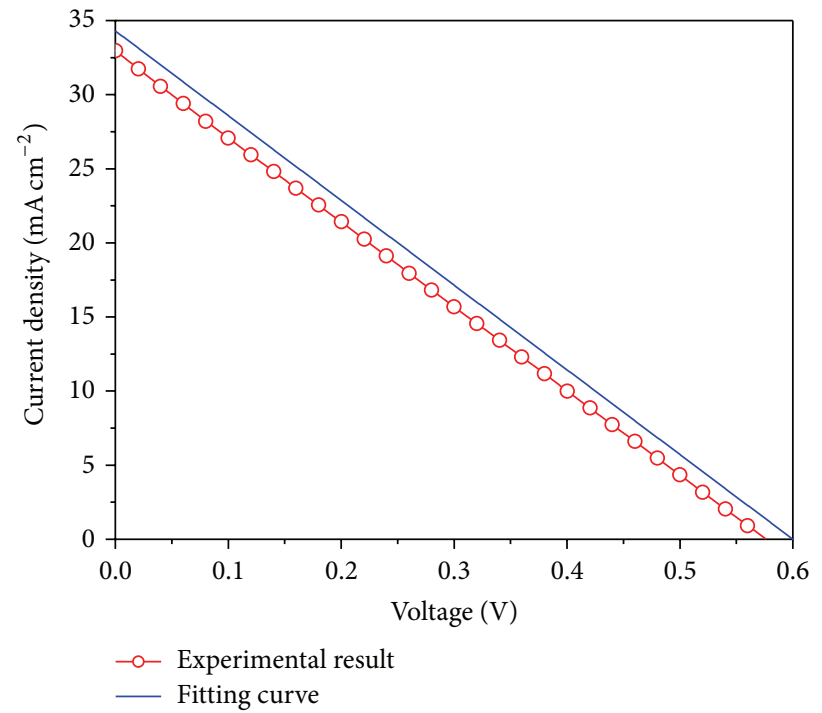

(b)

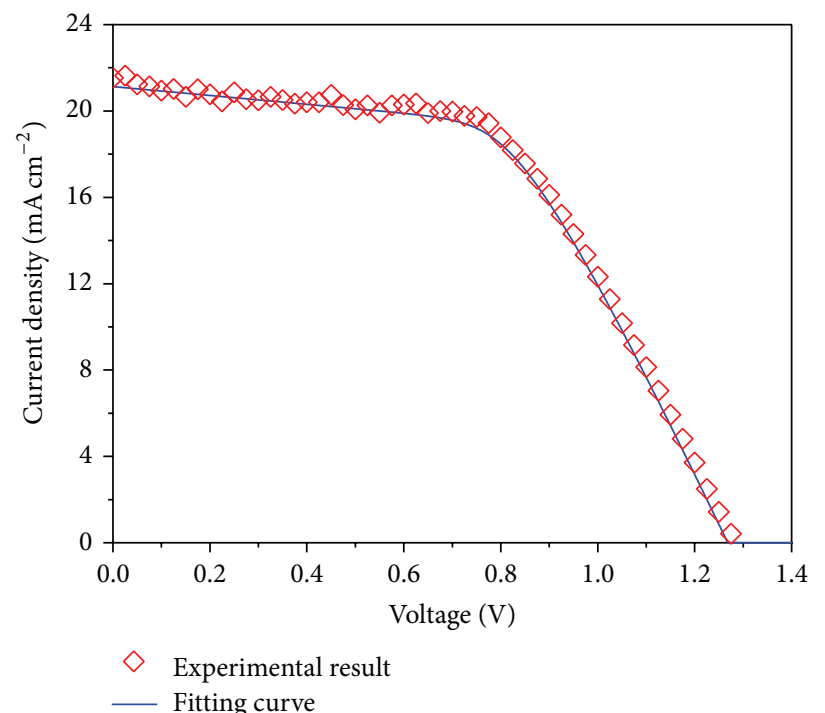

(c)

FIGURE 2: The experimental and fitting $J-V$ curves of (a) single DSSC, (b) single TE, (c) series DSSC, and TE hybrid device.

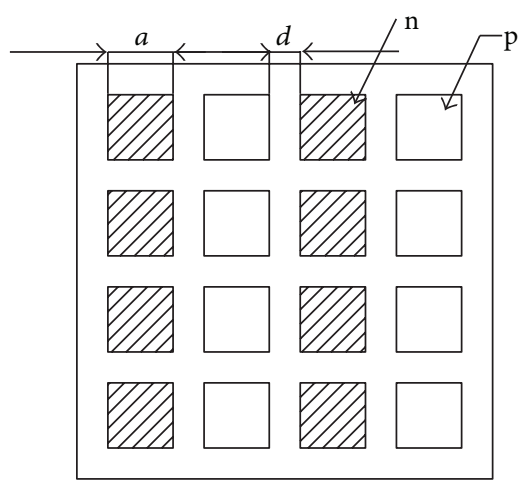

(a)

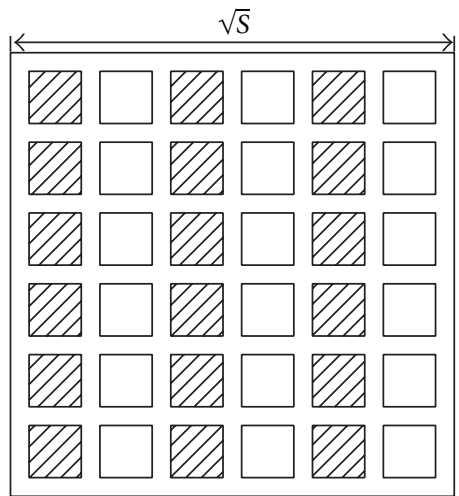

(b)

FIGURE 3: The top view of the TE p-n junctions array schematic diagram. 


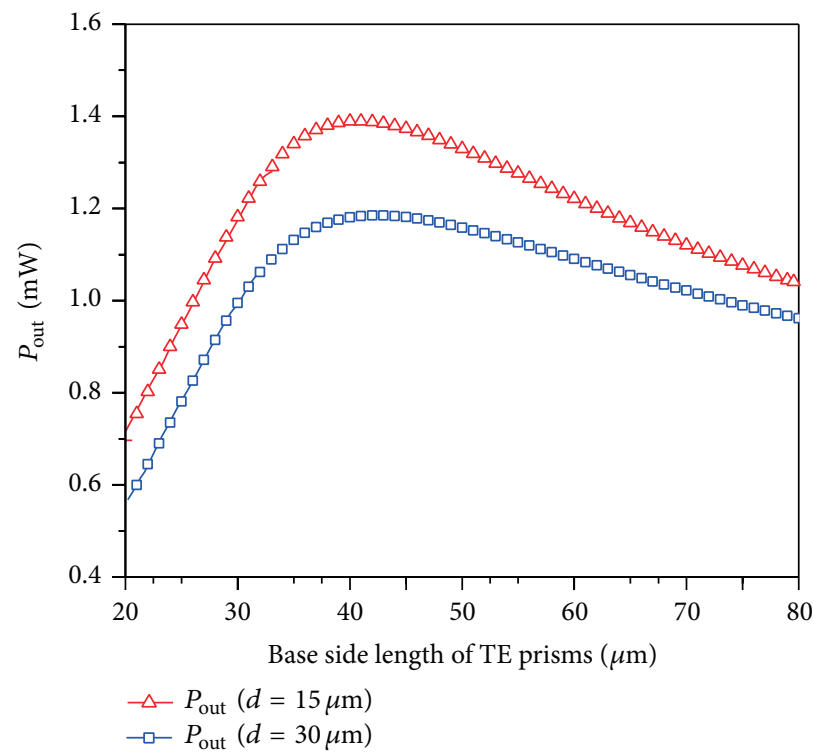

FIGURE 4: The effects of internal structure parameters $(a$ and $d$ ) in the TE modules on the output power of hybrid device.

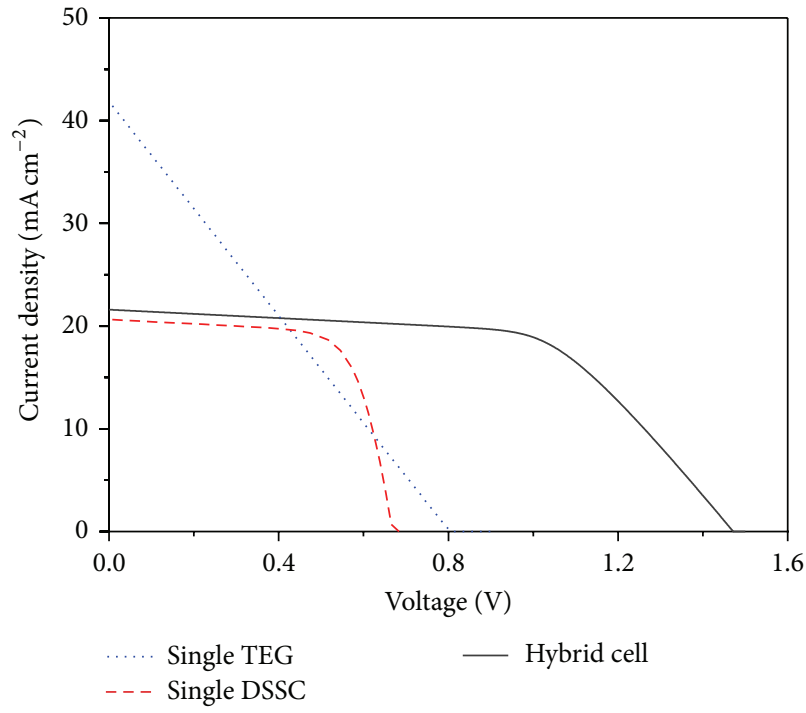

(a)

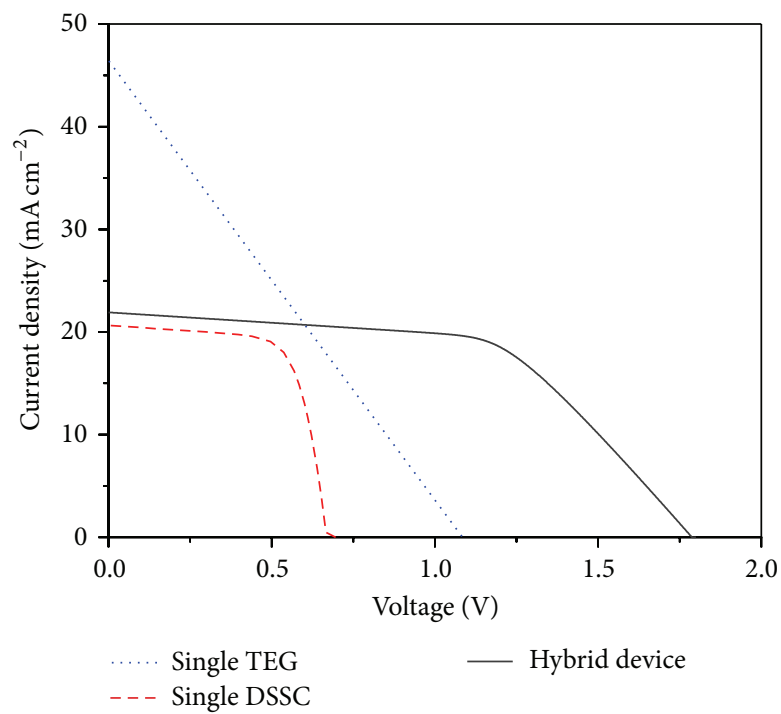

(b)

FIgure 5: The $J-V$ curves of TEG, DSSC, and hybrid device under simulated AM 1.5 solar spectrum irradiation at $100 \mathrm{~mW}^{-2}$ with (a) $d=15 \mu \mathrm{m}$ and (b) $d=30 \mu \mathrm{m}$.

a corresponding photoelectric conversion efficiency of the PV-TE hybrid device $18.96 \%$ as shown in Figure 5(a). This result is better than our previous experimental result [7]. If $d$ is reduced to $15 \mu \mathrm{m}$, the optimum $a$ is $40 \mu \mathrm{m}$ and the optimum output power reaches $1.389 \mathrm{~mW}$ with the corresponding efficiency $24.6 \%$ as shown in Figure 5(b), which is higher than that of the hybrid device with $d=30 \mu \mathrm{m}$. When $d$ decreases from $30 \mu \mathrm{m}$ to $15 \mu \mathrm{m}$, the TEG can accommodate more TE p$\mathrm{n}$ junctions, which converts more thermal energy into electric energy resulting in a higher efficiency. It can be expected that a smaller $d$ would cause a higher output power of TEG, but it would increase the difficulty of processing.

\section{Conclusions}

In summary, the photovoltaic-thermoelectric hybrid device is analyzed by the equivalent circuit method, and the internal structure size effect of TE modules on the properties of hybrid devices is investigated. Our preliminary simulational results demonstrate that the PV-TE hybrid device is anticipated for a much higher solar energy utilization efficiency than our previously reported experimental results by optimizing internal structure sizes of TE modules. When the spacing between TE prisms is $15 \mu \mathrm{m}$ and the optimal base side length of TE prism is $40 \mu \mathrm{m}$, the maximum theoretical efficiency reaches 
$24.6 \%$ according to the equivalent circuit analysis. This work gives some good enlightenment for the development of high-performance PV-TE hybrid devices. Meanwhile, this work fixes the temperature difference between the two sides of TEG and reckons without contact resistance of the TE modules in order to simplify the calculation, so there is still considerable room for further improvement of the hybrid devices and more efforts should be made to the simulational and experimental research on the high-performance PV-TE hybrid system.

\section{Conflict of Interests}

The authors declare that they have no conflict of interests regarding the publication of this paper.

\section{Acknowledgments}

This work was supported by Natural Science Foundation of China (nos. 51272035, 51272037, and 51362026), International Cooperation MOST-JST Program Fund (no. 2010DFA61410), the Project of International Cooperation of the Ministry of Science and Technology of China (no. 2011DFA50530), and Scientific Research Project of Guangdong (no. 2012B091000015).

\section{References}

[1] B. O'Regan and M. Grätzel, "A low-cost, high-efficiency solar cell based on dye-sensitized colloidal $\mathrm{TiO}_{2}$ films," Nature, vol. 353, no. 6346, pp. 737-740, 1991.

[2] A. Hagfeldt, G. Boschloo, L. Sun, L. Kloo, and H. Pettersson, "Dye-sensitized solar cells," Chemical Reviews, vol. 110, no. 11, pp. 6595-6663, 2010.

[3] H. Lin, X. Li, Y. Liu, and J. Li, "Progresses in dye-sensitized solar cells," Materials Science and Engineering B: Solid-State Materials for Advanced Technology, vol. 161, no. 1-3, pp. 2-7, 2009.

[4] P. Liska, K. R. Thampi, M. Grätzel et al., "Nanocrystalline dye-sensitized solar cell/copper indium gallium selenide thinfilm tandem showing greater than $15 \%$ conversion efficiency," Applied Physics Letters, vol. 88, no. 20, Article ID 203103, 2006.

[5] A. Nattestad, A. J. Mozer, M. K. R. Fischer et al., "Highly efficient photocathodes for dye-sensitized tandem solar cells," Nature Materials, vol. 9, no. 1, pp. 31-35, 2010.

[6] M. Yanagida, N. Onozawa-Komatsuzaki, M. Kurashige, K. Sayama, and H. Sugihara, "Optimization of tandem-structured dye-sensitized solar cell," Solar Energy Materials and Solar Cells, vol. 94, no. 2, pp. 297-302, 2010.

[7] N. Wang, L. Han, H. He, N.-H. Park, and K. Koumoto, "A novel high-performance photovoltaic-thermoelectric hybrid device," Energy and Environmental Science, vol. 4, no. 9, pp. 3676-3679, 2011.

[8] A. Amine, Y. Mir, and M. Zazoui, "Modelling of dual-junction solar cells including tunnel junction," Advances in Condensed Matter Physics, vol. 2013, Article ID 546362, 5 pages, 2013.

[9] L. Han, N. Koide, Y. Chiba, A. Islam, and T. Mitate, "Modeling of an equivalent circuit for dye-sensitized solar cells: improvement of efficiency of dye-sensitized solar cells by reducing internal resistance," Comptes Rendus Chimie, vol. 9, no. 5-6, pp. 645-651, 2006.
[10] M. R. Mitroi, L. Fara, and M. L. Ciurea, "Numerical procedure for optimizing dye-sensitized solar cells," Journal of Nanomaterials, vol. 2014, Article ID 378981, 6 pages, 2014.

[11] M. Murayama and T. Mori, "Novel tandem cell structure of dye-sensitized solar cell for improvement in photocurrent," Thin Solid Films, vol. 516, no. 9, pp. 2716-2722, 2008.

[12] M. Murayama and T. Mori, "Evaluation of treatment effects for high-performance dye-sensitized solar cells using equivalent circuit analysis," Thin Solid Films, vol. 509, no. 1-2, pp. 123-126, 2006.

[13] B. S. Yilbas and A. Z. Sahin, "Thermoelectric device and optimum external load parameter and slenderness ratio," Energy, vol. 35, no. 12, pp. 5380-5384, 2010.

[14] D. Kraemer, B. Poudel, H.-P. Feng et al., "High-performance flat-panel solar thermoelectric generators with high thermal concentration," Nature Materials, vol. 10, no. 7, pp. 532-538, 2011. 

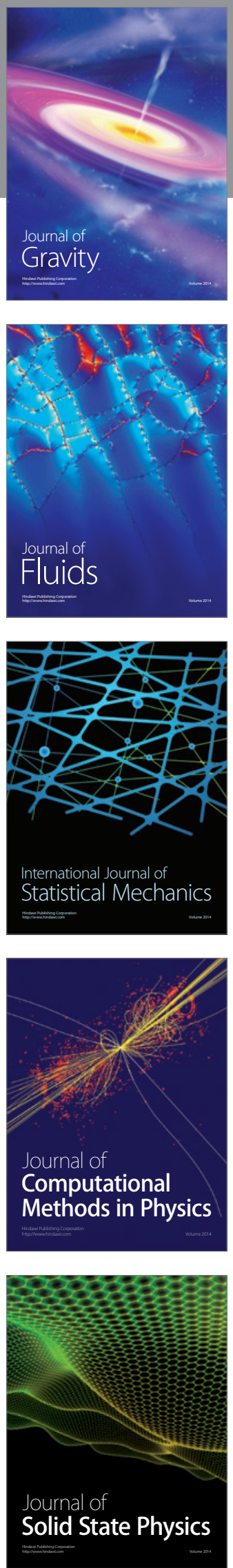

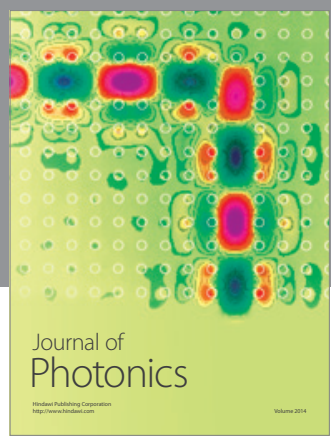

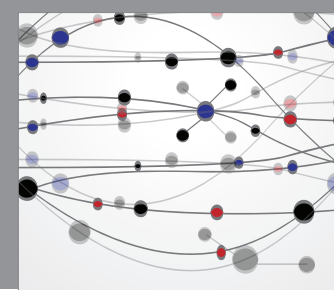

The Scientific World Journal

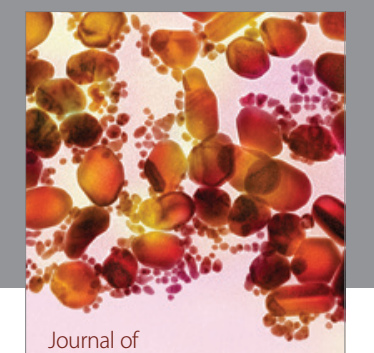

Soft Matter
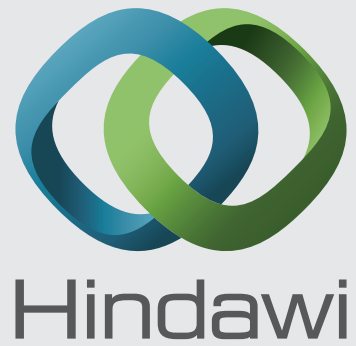

Submit your manuscripts at

http://www.hindawi.com
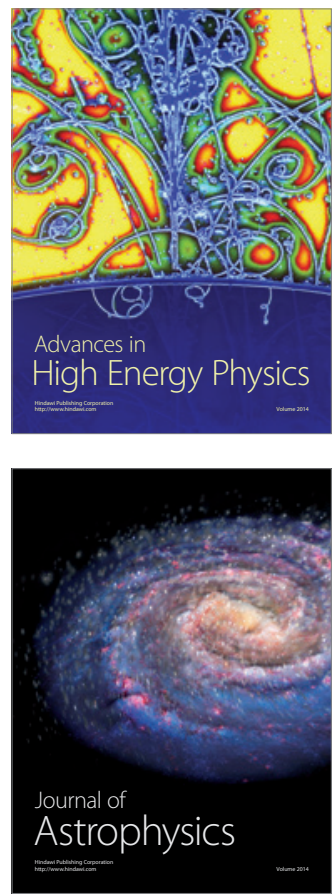
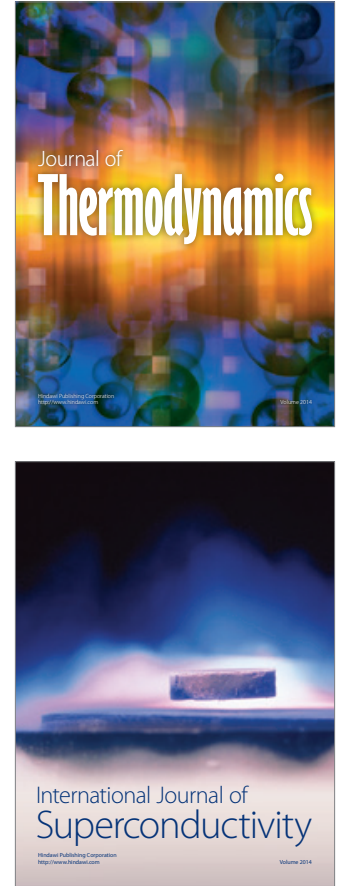
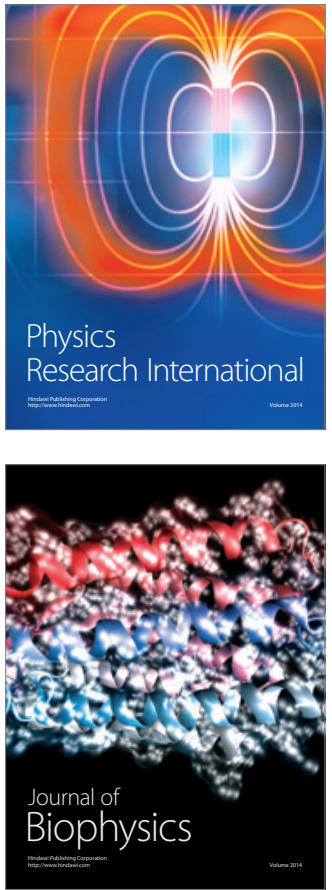
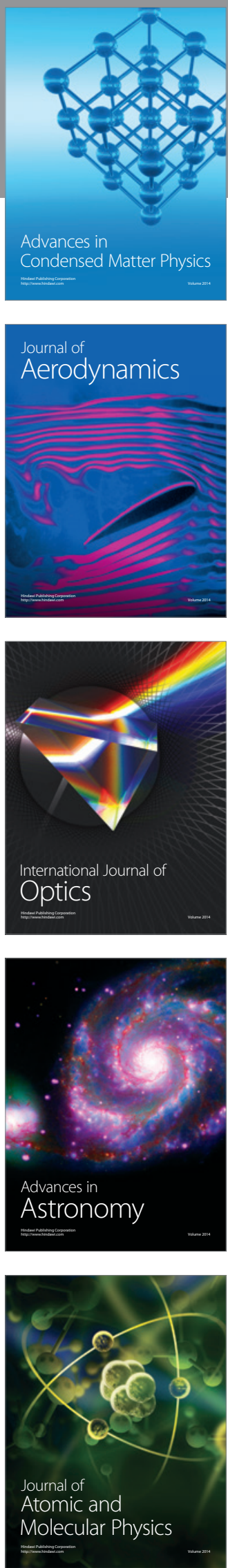